\title{
Serum miRNA expression in patients with esophageal squamous cell carcinoma
}

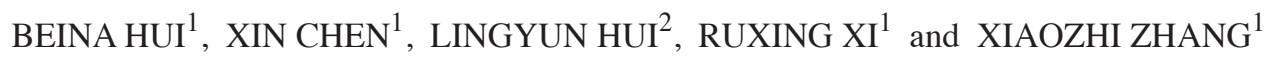 \\ Departments of ${ }^{1}$ Radiation Oncology and ${ }^{2}$ Clinical Laboratory, \\ First Affiliated Hospital of Xi'an Jiaotong University School of Medicine, Xi'an, Shaanxi 710061, P.R. China
}

Received October 20, 2014; Accepted July 17, 2015

DOI: 10.3892/ol.2015.3642

\begin{abstract}
The aim of the present study was to investigate the feasibility and value of serum microRNAs (miRNAs/miRs) as biological markers for the prediction of the behavior and prognosis of esophageal squamous cell cancer (ESCC). The differential expression of serum miRNA was detected by an miRNA microarray of 9 patients with ESCC and 9 healthy volunteers. The result of the miRNA microarray was validated in serum samples of 69 patients with ESCC and 14 healthy volunteers by reverse transcription-quantitative polymerase chain reaction (RT-qPCR). The association between serum miRNA expression and tumor-node-metastasis (TNM) stage was analyzed. A total of 10 serum-specific miRNAs were identified from the patients with ESCC. Through PCR verification, the expression levels of miR-129, miR-451 and miR-365 were consistent with the microarray results validated by RT-qPCR, and the difference was statistically significant compared with the healthy volunteers $(\mathrm{P}=0.007, \mathrm{P}=0.007$ and $\mathrm{P}<0.001$, respectively). Multivariate logistic regression analysis showed that miR-365 could serve as potential diagnostic marker for ESCC; the area under the receiver operating characteristic curve was 0.831 , with a sensitivity of $80.56 \%$ and a specificity of $86.7 \%$, but its expression did not differ significantly among the different TNM stages (stage I-II vs. III, $\mathrm{P}=0.052$; stage III vs. IV, $\mathrm{P}=0.069)$. The expression level of miRNA-129 differed significantly among the different stages (stage I-II vs. III, , $\mathrm{P}=0.002$; stage III vs. IV, $\mathrm{P}=0.042$ ), while the expression level of miR-451 did not differ significantly between stage III and IV $(\mathrm{P}=0.308)$. In conclusion, serum microRNAs are novel biomarkers for ESCC, and miRNA-365 and miRNA-129 can be used for the early prediction of cancer and the prediction of clinical stage, respectively.
\end{abstract}

Correspondence to: Professor Xiaozhi Zhang, Department of Radiation Oncology, First Affiliated Hospital of Xi'an Jiaotong University School of Medicine, 277 Yanta West Road, Xi'an, Shaanxi 710061, P.R. China

E-mail: zhangxiaozhimail@163.com

Key words: esophageal squamous cell cancer, serum, microRNA, biological markers

\section{Introduction}

Esophageal cancer is one of the most common malignant tumors in China. Each year there are 288,000 new cases, leading to 208,000 fatalities; squamous cell carcinoma (SCC) accounts for $90 \%$ of these cases (1). Esophageal cancer is also characterized by an insidious onset. The majority of patients are already at an advanced stage when they are first diagnosed, and the 5-year survival rate following surgery is only $25 \%$. However, at an early disease stage, the 5-year survival rate following surgery is as high as $80-90 \%$ (2). Therefore, early diagnosis and treatment are crucial. Existing examination methods for diagnosing esophageal SCC (ESCC), including esophageal cytology, X-ray, computed tomography examination and endoscopic ultrasound, cannot achieve an early diagnosis (3). It is abundantly clear that finding the ideal ESCC marker with a high specificity and sensitivity is a pressing issue.

microRNAs (miRNAs/miRs) are small non-coding RNAs of 19-24 nucleotides in length that are involved in cancer development (4). Recent studies have shown that miRNA expression is stable enough to be detectable in the blood and to serve as a useful cancer biomarker. For example, it has been shown that it is possible to improve the potential early diagnosis and treatment of ovarian cancer lacking clinical signs. It was demonstrated that the expression levels of miR-21, miR-92, miR-93, miR-126 and miR-29a in the serum of ovarian cancer patients were significantly upregulated, whereas those of miR-155, miR-127 and miR-99b were significantly reduced $(5,6)$. In patients with diffuse large B-cell lymphoma, the serum miR-21 expression level was significantly increased, and the upregulation of miR-21 expression was also correlated with a higher recurrence-free survival rate $(7,8)$. These results suggest that the detection of the specific expression of miRNAs not only assists in the early diagnosis of cancer, but also in the determination of the patient's prognosis. These features point to the superior application of miRNAs as biological markers.

Therefore, in the present study, the Agilent MicroRNA Microarray Profiling System and bioinformatics methods were used for the analysis and identification of specific miRNAs in the serum of ESCC patients. Reverse transcription-quantitative polymerase chain reaction (RT-qPCR) was applied to verify the microarray results. The goal of the study was to investigate the feasibility and value of serum microRNAs as biological 
markers for the prediction of the biological behavior and prognosis of ESCC.

\section{Patients and methods}

Participants. Peripheral blood samples were collected from 78 esophageal cancer patients at the First Affiliated Hospital of Xi'an Jiaotong University School of Medicine (Xi'an, Shaanxi, China) between June 2012 and December 2013. None of the patients had received any treatments prior to sample collection, including surgery, radiotherapy or chemotherapy. All cases were pathologically confirmed as ESCC, and none had a history of any other type of cancer. The cohort consisted of 57 males and 21 females, with ages ranging from 53-79 years and a mean age of 58.55 years. Blood samples were also collected from 23 age and gender matched healthy volunteers. All samples were collected after obtaining informed consent from the participant. Written informed consent was obtained from all patients prior to the study, and the study was approved by the Ethics Committee of the First Affiliated Hospital of Xi'an Jiaotong University.

Collection of serum samples. In each case, $2 \mathrm{ml}$ of collected peripheral blood was quickly transferred to an ordinary EDTA-free tube, and allowed to settle at room temperature $\left(22-25^{\circ} \mathrm{C}\right)$ for $15-30 \mathrm{~min}$, until completely coagulated. Next, 0.6-1 $\mathrm{ml}$ of supernatant was aspirated and transferred to a clean 1.5-ml centrifuge tube, and the following steps were completed within $1 \mathrm{~h}$ (at room temperature) or $2 \mathrm{~h}$ (at $4^{\circ} \mathrm{C}$ ): Centrifugation at $820 \mathrm{x} \mathrm{g}$ for $10 \mathrm{~min}$ at $4^{\circ} \mathrm{C}$; collection of supernatant and transferal to a clean 1.5-ml centrifuge tube; centrifugation at $16,000 \mathrm{x}$ g for $10 \mathrm{~min}$ at $4^{\circ} \mathrm{C}$; aspiration of the supernatant and transferal to another clean centrifuge tube; and storage at $-80^{\circ} \mathrm{C}$ for future use.

Extraction of total RNA from serum. The serum samples prepared as aforementioned were collected (400 $\mu \mathrm{l}$ each) and total RNA was extracted from the serum using the mirVana ${ }^{\mathrm{TM}}$ PARIS $^{\text {TM }}$ kit (Applied Biosystems Life Technologies, Foster City, CA, USA) according to the manufacturer's instructions. RNA ( $1 \mu \mathrm{l})$ was used for quantitative analysis using a NanoDrop ND-2000 (Thermo Fisher Scientific, Inc., Waltham, MA, USA), and an Agilent Bioanalyzer 2100 (Agilent Technologies Inc., Santa Clara, CA, USA) was used to examine RNA integrity.

Detection of serum miRNA expression using the Agilent MicroRNA Microarray Profiling System. Total RNA (100 ng) was extracted from the sera of 9 ESCC patients and 9 healthy volunteers, and used for dephosphorylation, Cy3 labeling and hybridization with a miRNA microarray using the miRNA Complete Labeling and Hyb kit (Agilent Technologies Inc.), according to the manufacturer's instructions. Following hybridization, the microarray was washed with the washing buffer in the Gene Expression Wash Buffer kit (Agilent Technologies Inc.. Finally, an Agilent G2505C Scanner (Agilent Technologies Inc.) was used to scan the microarray, and GeneSpring GX11.0 software (Agilent Technologies Inc.) was used for signal processing. The Quantile method was applied for data normalization.
Table I. Differentially-expressed miRNAs $(\mathrm{P}<0.01)$ in esophageal squamous cell carcinoma patients with $>8$-fold change selected from microarray data analysis.

\begin{tabular}{lcrc}
\hline Name & P-value & Fold-change & Regulation \\
\hline hsa-miR-365 & 0.001805756 & 8.365508 & $\mathrm{Up}$ \\
hsa-miR-92b & 0.002613297 & 15.273841 & $\mathrm{Up}$ \\
hsa-miR-602 & 0.005954986 & 13.051751 & $\mathrm{Up}$ \\
hsa-miR-518b & 0.004742492 & 9.271833 & Down \\
hsa-miR-767-3p & 0.007218791 & 10.837473 & $\mathrm{Up}$ \\
hsa-miR-451 & 0.001277682 & 15.483809 & $\mathrm{Up}$ \\
hsa-miR-563 & 0.001073018 & 12.695153 & $\mathrm{Up}$ \\
hsa-miR-129 & 0.001711119 & 9.499698 & $\mathrm{Up}$ \\
hsa-miR-18b* & 0.003863498 & 8.473071 & $\mathrm{Up}$ \\
hsa-miR-548 & 0.002055900 & 9.823999 & $\mathrm{Up}$ \\
\hline
\end{tabular}

RT-qPCR for validation of microarray results. Total serum RNA (60 ng) from the remaining 69 ESCC patients and 14 healthy volunteers was subjected to RT reactions using the TaqMan miRNA Reverse Transcription kit (Applied Biosystems Life Technologies). The reaction conditions were as follows: $30 \mathrm{~min}$ at $16^{\circ} \mathrm{C}, 30 \mathrm{~min}$ at $42^{\circ} \mathrm{C}$ and $5 \mathrm{~min}$ at $85^{\circ} \mathrm{C}$; the reaction preparation was then maintained at $4^{\circ} \mathrm{C}$. Specific fluorescent TaqMan Probe was used for the RT-qPCR assay to detect the plasma miRNA content. In accordance with previous studies, miR-1228 was used as the internal control $(9,10)$. The PCR conditions were as follows: $96^{\circ} \mathrm{C}$ for $5 \mathrm{~min}$, followed by 50 cycles at $95^{\circ} \mathrm{C}$ for $15 \mathrm{sec}$, and then $60^{\circ} \mathrm{C}$ for $1 \mathrm{~min}$. The relative expression level of each miRNA was expressed using the $2^{-\Delta \Delta \mathrm{Ct}}$ method, where $\Delta \mathrm{Ct}=\mathrm{Ct}_{\text {miRNA }}-\mathrm{Ct}_{\text {mi1228 }}$. Each experiment was repeated three times.

Statistical methods. GeneSpring GX 11.0 software was used to analyze the microarray data. Fold-change analysis and t-tests were performed for differential gene expression analysis (screening criteria, fold-change $>2 ; \mathrm{P}<0.01$ ). MedCalc software was used for the Mann-Whitney $U$ and Kruskal-Wallis tests of the quantitative fluorescence, qPCR results. The receiver operator characteristic curves (ROC) were used to evaluate the sensitivity and specificity of the expression levels of different miRNAs in the diagnosis of ESCC. Multivariate logistic regression analysis was performed to find the optimal combination of miRNAs. $\mathrm{P}<0.05$ was considered to indicate a statistically significant difference.

\section{Results}

Serum miRNA expression, as detected by miRNA microarray. A total of 42 ESCC-specific serum miRNAs were obtained from the miRNA microarray results using the screening criteria of a fold-change of $>2$ and $\mathrm{P}<0.05$ for the t-test. To improve the likelihood of differential gene expression, the screening criteria were increased to a fold-change of $>8$ and $\mathrm{P}<0.01$ for the t-test; a total of $10 \mathrm{ESCC}$-specific serum miRNAs were then obtained (Table I). 
A

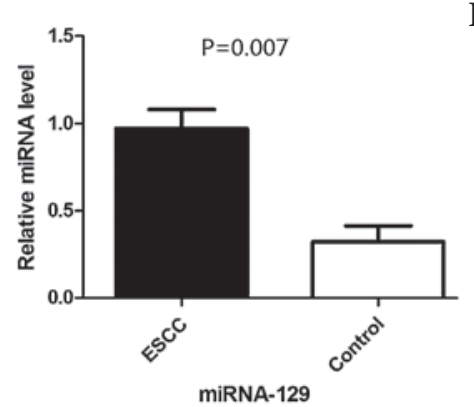

B

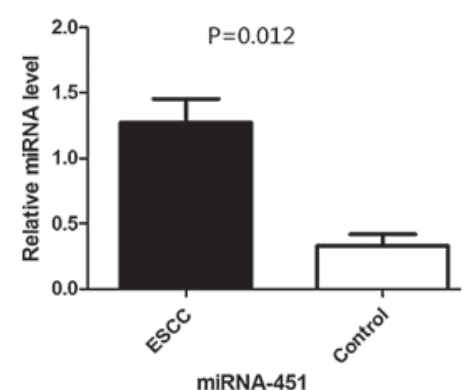

C

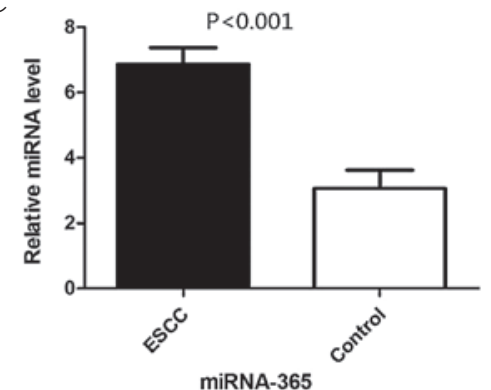

Figure 1. Expression levels of significantly elevated microRNAs (miRNAs/miRs), (A) miR-129, (B) miR-451 and (C) miR-365, in serum from patients with esophageal squamous cell carcinoma, as detected by reverse transcription-quantitative polymerase chain reaction.

A

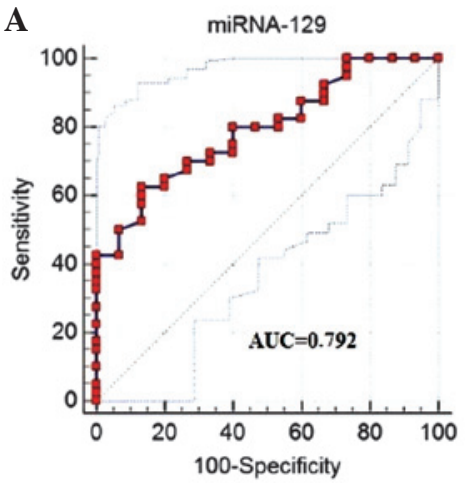

B

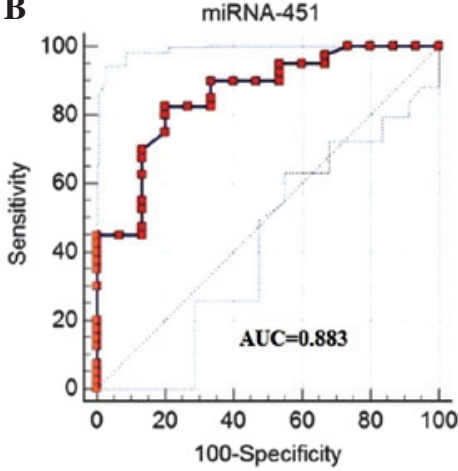

C

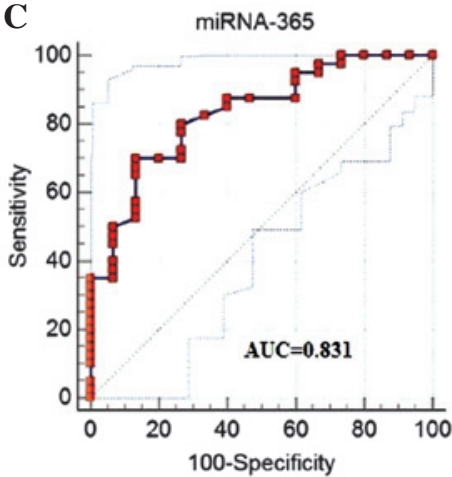

Figure 2. Receiver operating characteristic curves of serum (A) miR-129, (B) miR-451 and (C) miR-365. miRNA/miR, microRNA; AUC, area under the curve.

Validation of microarray results using RT-qPCR. RT-qPCR showed consistent results with regard to the expression levels of miR-129, miR-451 and miR-365, and the microarray results; in addition, compared with the healthy controls, the expression levels of these miRNAs were significantly different $(\mathrm{P}=0.007$, $\mathrm{P}=0.012$ and $\mathrm{P}<0.001$, respectively) (Fig. 1). For miR-92b, miR-563 and miR-767-3p, although the directionality of the changes in the expression levels was consistent with the microarray results, the expression levels of these miRNAs were not significantly different from that of the healthy volunteers ( $\mathrm{P}>0.05$ ). The miR-518b and miR-548 expression levels, as detected with quantitative fluorescence qPCR, were inconsistent with those obtained from the microarray analysis. Due to poor specificity of the melting curve, data on the expression levels of miR-602 and miR-18b* was not obtained.

Value of miR-129, miR-451 and miR-365 in ESCC diagnosis. The ROC analysis results showed that by using the relative expression of miR-129 alone (cutoff, 0.386), the sensitivity was $78.8 \%$, the specificity was $73.3 \%$ and the area under the curve (AUC) was 0.792 . Using the relative expression of miR-451 alone (cutoff, 0.568), the sensitivity was $82.5 \%$, the specificity was $79.0 \%$ and the AUC was 0.882 . Using the relative expression of miR-365 alone (cutoff value, 5.06), the sensitivity for ESCC diagnosis was $80.56 \%$ and the specificity was $86.7 \%$, with an AUC of 0.831 (Fig. 2). Multivariate logistic regression analysis showed that miR-365 could serve as a potential diagnostic marker for $\operatorname{ESCC}(\mathrm{P}=0.002)$, but that adding miR-451 or miR-129 could not further improve the diagnostic efficiency.
Correlation between serum miRNA expression levels and TNM stage. It was found that even in the early stages of ESCC, miR-365 expression was already significantly elevated compared with the healthy controls (stage I-II, $\mathrm{P}=0.025$; stage III, $\mathrm{P}=0.001$; stage $\mathrm{IV}, \mathrm{P}=0.001$; Mann-Whitney $\mathrm{U}$ test); however, its expression did not differ significantly among different TNM stages (stage II vs. III, $\mathrm{P}=0.452$; stage III vs. IV, $\mathrm{P}=0.389$ ). The miR-451 and miR-129 expression levels did not increase significantly compared with the normal controls in early-stage ESCC, but did increase significantly at stages III and IV. In particular, the expression level of miRNA-129 differed significantly among different stages (stage II vs. III, $\mathrm{P}=0.002$; stage III vs. IV, $\mathrm{P}=0.042$ ). However, the expression level of miR-451 did not differ significantly between stages III and IV (stage II vs. III, $\mathrm{P}=0.004$; stage III vs. IV, $\mathrm{P}=0.308$ ) (Fig. 3).

\section{Discussion}

The present study examined and analyzed the serum miRNA expression profile of ESCC patients using miRNA microarray technology and subsequent validation with quantitative fluorescence-based, qPCR. It was found that the expression of 3 miRNAs, miR-129, miR-451 and miR-365, was significantly elevated compared with the healthy controls. Further multivariate regression analysis revealed that miR-365 can serve as a biomarker for the early diagnosis of ESCC, with a sensitivity of $80.56 \%$, a specificity of $86.7 \%$ and an AUC of 0.831 . The miR-365 expression level was significantly increased in the serum of TNM stage I and II ESCC patients, but such specific 

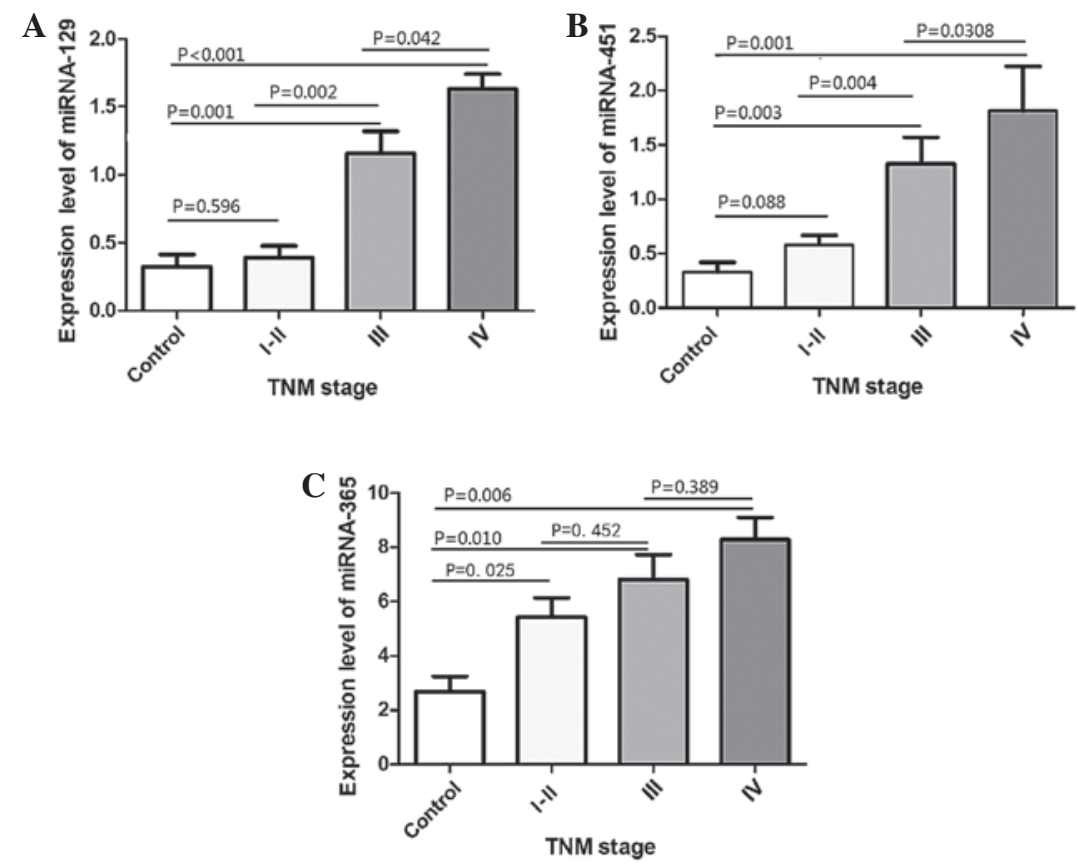

Figure 3. Expression levels of serum (A) miR-129, (B) miR-451 and (C) miR-365 from esophageal squamous cell carcinoma patients with different TNM stages. miR/miRNA, microRNA; TNM, tumor-node-metastasis.

upregulation was not correlated with the TNM stage; this suggested that miR-365 may not be a suitable indicator of ESCC progression. The upregulation of miR-451 and miR-129 in the early stages of ESCC was not significant, but became statistically significant at stages III and IV. In particular, the expression level of miRNA-129 differed significantly between the different stages, suggesting that it may serve as an independent monitoring indicator for the prognosis of ESCC.

To date, there have no studies on the expression of the 3 miRNAs, miR-129, miR-451 and miR-365, in the serum of cancer patients. A recent study showed that circulating miRNAs were selectively secreted via a cellular selection mechanism and that this selection mechanism was associated with the degree of malignancy (11). It was also demonstrated that living cells secreted miRNAs capable of inhibiting each other (12). With regard to miR-365, overexpression was detected in these cells and the clinical specimens of cutaneous squamous cell carcinoma. In vivo experiments have shown that the HaCaTpre-miR-365-2 cell line, which overexpresses miR-365, can induce the formation of subcutaneous tumors, and that the use of the anti-miR-365 oligonucleotide Antagomir-365 can inhibit the formation of skin tumors in vivo, inducing tumor cell cycle arrest at the $G$ phase and apoptosis (13). These results suggest that miR-365 acts as an oncomiR in cutaneous squamous cell carcinoma in vitro and in vivo. In the present study, an elevation in the expression of miR-365 in the sera of stage I and II ESCC patients was found, which was possibly due to the fact that miR-365 is associated with the early formation of esophageal cancer. Ogawa et al (14) utilized RT-PCR to identify 7 miRNAs in ESCC cells, and found miRNA-129 overexpression in these cells. Analysis of the post-operative survival data subsequently showed that high levels of miRNA-129 were negatively correlated with the post-operative survival time of the patients, and multi-factor regression analysis also suggested that miRNA-129 was an independent prognostic factor. In the present study, a significant elevation in the expression of miRNA-129 in the serum from stage III and IV ESCC patients was observed, and it was deduced that a high expression level of miRNA-129 may be associated with the tumor burden, and that miRNA-129 could potentially serve as an independent factor for the prognostic monitoring of esophageal cancer. Recent studies have found that miR-451 is not only expressed abnormally in tumor tissues, but that it is also involved in the biological activity of certain tumors and the genetic regulation of their drug resistance (15-18). The current literature illustrates the downregulation of miR-451 in gastric, colon, esophageal and non-small-cell lung cancer, and its upregulation in head and neck squamous cell carcinoma and multidrug-resistant ovarian cancer (19-21). Wang et al found that the upregulation of miR-451 expression in EC9706 esophageal cancer cells effectively inhibited proliferation and invasiveness, and promoted apoptosis, possibly through the phosphoinositide 3-kinase/Akt signaling pathway (22). Therefore, we hypothesize that the body may produce miR-451 to inhibit the proliferation of advanced esophageal cancer cells, leading to an increase in miR-451 levels in the circulating blood.

It has been previously reported that miRNA-21 can serve as a marker for the diagnostic and prognostic monitoring of esophageal cancer (23-25). However, miRNA-21 expression was also shown to be elevated in other types of tumors and diseases, such as colorectal, lung and gastric cancer (26-28), and thus cannot in fact serve as a specific marker for esophageal cancer. In addition, there have been several studies on the esophageal cancer diagnosis using a combination of multiple miRNAs. For example, Zhang et al (29) applied Solexa sequencing to compare the serum miRNA expression profile of ESCC patients against that of healthy controls, and found that 25 miRNAs exhibited significantly elevated expression. Furthermore, 
RT-qPCR revealed significant increases in 7 miRNAs (miR-10a, $22,100,148 \mathrm{~b}, 223,133 \mathrm{a}$ and 127-3p), in the serum of the ESCC patients. Cluster analysis illustrated that the combination of these 7 miRNAs could distinguish $89.6 \%$ of early ESCC patients from normal controls, showing miRNAs to be of relatively significant value in the early diagnosis of ESCC (29). The present study failed to replicate the results of previous studies; that this may be due to differences in experimental design, material selection and detection techniques between the studies. In the current study, the relevant miRNAs were screened and identified from an miRNA microarray containing 2,026 miRNAs, and these were then validated using RT-qPCR. Hence, the present results should be quite reliable. Thus, these miRNAs exhibit a relatively high importance in terms of their research value.

Certain limitations must be considered when interpreting the results of the present study. First, the sample size was small. Second, the abundance of the miRNAs in the serum was too low to be accurately quantified by RT-qPCR, therefore, certain potential relevant markers could not be assessed. Third, there have been a few studies on the expression of miR-365, miR-451 and miR-129 in the serum or on the mechanisms subserving their functions, and this remains unclear. Therefore, in the next stage of our studies, the sample size will be expanded and complete follow-up examinations of the patients will be performed so as to understand the association between miRNA expression and patient survival. In addition, future studies will characterize the expression of these miRNAs in ESCC tissues and cells, and investigate the mechanisms underlying their functions in ESCC. Such studies should aid in the determination of the reason for their elevation in the serum.

In conclusion, the present study found that the expression levels of miR-129, miR-451 and miR-365 were significantly increased in the serum of ESCC patients compared with healthy subjects. Specifically, we propose that miR-365 be used as a potential marker for early ESCC diagnosis, while miRNA-129, which showed expression levels associated with tumor stage, may serve as an ESCC prognostic indicator. For the purposes of the early detection and diagnosis of ESCC, and to improve patient survival, larger studies must be performed to screen and verify combinations of ESCC-specific serum biomarkers; these markers could then be used as a sensitive, specific, non-invasive and simple method for tumor detection in the early diagnosis and prognostic monitoring of ESCC.

\section{Acknowledgements}

This study was financially supported by the National Natural Science Foundation of China (grant no. 30972962).

\section{References}

1. Chen W, Zheng R, Zhang S, Zhao P, Zeng H, Zou X and He J: Annual report on status of cancer in China, 2010. Chin J Cancer Res 26: 48-58, 2014.

2. Wang GQ, Jiao GG, Chang FB, et al: Long-term results of operation for 420 patients with early squamous cell esophageal carcinoma discovered by screening. Ann Throac surg 77: 1740-1744, 2004.

3. Campbell F, Lauwers GY and Williams GT: Tumors of the esophagus and stomach. In: Diagnostic Histopathology of Tumors. Fletcher CDM (ed). 3rd edition. Churchill Livingstone Elsevier, London, pp328-329, 2007.

4. Bartel DP: MicroRNAs: Genomics, biogenesis, mechanism and function. Cell 116: 281-297, 2004.
5. Resinick KE, Alder H, Hagan JP, et al: The detection of differentially expressed microRNA from the serum of ovarian cancer patients using a novel real-time PCR platform. Gynecol Oncol 112: 55-59, 2009.

6. Taylor DD and Gercel-Taylor C: MicroRNA signatures of tumor-derived exosomes as diagnostic biomarkers of ovarian cancer. Gynecol Oncol 110: 13-21, 2008.

7. Lawrie CH, Gal S, Dunlop HM, et al: Detection of elevated levels of tumor-associated microRNAs in serum of patients with diffuse large B-cell lymphoma. Br J Haematol 141: 672-675, 2008.

8. Chin LJ and Slack FJ: A truth serum for cancer-microRNAs have major potential as cancer biomarkers. Cell Res 18: 983-984, 2008.

9. Zhou J, Yu L, Gao X, et al: A Plasma MicroRNA panel to diagnose hepatitis B virus related hepatocellular carcinoma. J Clin Oncol 29: 4781-4788, 2011.

10. Hu J, Wang Z, Liao BY, et al: Human miR-1228 as a stable endogenous control for the quantification of circulating microRNAs in cancer patients. Int J Cancer 135: 1187-1194, 2014.

11. Pigati L, Yaddanapudi SC, Iyengar R, Kim DJ, Hearn SA, Danforth D, Hastings ML and Duelli DM: Selective release of microRNA species from normal and malignant mammary epithelial cells. PLoS One 5: e13515, 2010.

12. Iguchi H, Kosaka N and Ochiya T: Secretory microRNAs as a versatile communication tool. Commun Integr Biol 3: 478-481, 2010.

13. Zhou M, Liu W, Ma S, et al: A novel onco-miR-365 induces cutaneous squamous cell carcinoma. Carcinogenesis 34: 1653-1659, 2013

14. Ogawa R, Ishiguro H, Kuwabara Y, et al: Expression profilinf of micro-RNAs in human esophageal squamous cell carcinoma using RT-PCR. Med Mol Morphol 42: 102-109, 2009.

15. Pan X, Wang R and Wang ZX: The potential role of miR-451 in cancer diagnosis, prognosis, and therapy. Mol Cancer Ther 12: 1153-1162, 2013.

16. Kovalchuk O, Filkowski J, Meservy J, et al: Involvement of microRNA-451 in resistance of the MCF-7 breast cancer cells to chemotherapeutic drug doxorubicin. Mol Cancer Ther 7: 2152-2159, 2008.

17. Zhu H, Wu H, Liu X, et al: Role of MicroRNA miR-27a and miR-451 in the regulation of MDR1/P-glycoprotein expression in human cancer cells. Biochem Pharmacol 76: 582-588, 2008.

18. Wang XC, Tian LL, Jiang XY, et al: The expression and function of miRNA-451 in non-small cell lung cancer. Cancer Lett 311: 203-209, 2011.

19. Banders E, Bitarte N, Arias F, et al: MicroRNA-451 regulates macrophage migration inhibitory factor production and proliferation of gastrointestinal cancer cells. Clin Cancer Res 15: 2281-2290, 2009.

20. Wang R, Wang ZX, Yang JS, Pan X, De W and Chen LB: MicroRNA-451 functions as a tumor suppressor in human non-small cell lung cancer by targeting ras-related protein 14 (RAB14). Oncogene 30: 2644-2658, 2011.

21. Hui AB, Lenarduzzi M, Krushel $\mathrm{T}$, et al: Comprehensive MicroRNA profiling for head and neck squamous cell carcinomas. Clin Cancer Res 16: 1129-1139, 2010.

22. Wang T, Zang WQ, Li M, Wang N, Zheng YL and Zhao GQ: Effect of miR-451 on the biological behavior of esophageal carcinoma cell line EC9706. Dig Dis Sci 58: 706-714, 2013.

23. Tanaka Y, Kamohara H, Kinoshita K, et al: Clinical impact of serum exosomal microRNA-21 as a clinical biomarker in human esophageal squamous cell carcinoma. Cancer 119: 1159-1167, 2013.

24. Cai EH, Gao YX, Wei ZZ, Chen WY, Yu P and Li K: Serum miR-21 expression in human esophageal squamous cell carcinomas. Asian Pac J Cancer Prev 13: 1563-1567, 2012.

25. KurashigeJ,KamoharaH,WatanabeM, et al: Serum microRNA-21 is a novel biomarker in patients with esophageal squamous cell carcinoma. J Surg Oncol 106: 188-192, 2012.

26. Basati G, Emami Razavi A, Abdi S and Mirzaei A: Elevated level of microRNA-21 in the serum of patients with colorectal cancer. Med Oncol 31: 205, 2014

27. Yang X, Guo Y, Du Y, et al: Serum microRNA-21 as a diagnostic marker for lung carcinoma: A systematic review and meta-analysis. PLoS One 9: e97460, 2014.

28. Song J, Bai Z, Zhang J, et al: Serum microRNA-21 levels are related to tumor size in gastric cancer patients but cannot predict prognosis. Oncol Lett 6: 1733-1737, 2013.

29. Zhang C, Wang C, Chen X, et al: Expression profile of microRNAs in serum: A fingerprint for esophageal squamous cell carcinoma. Clin Chem 56: 1871-1879, 2010. 